\title{
Rotational chaos and strange attractors on the 2-torus
}

\author{
Jan P. Boroński · Piotr Oprocha
}

Received: 13 December 2013 / Accepted: 15 September 2014 / Published online: 31 October 2014

(C) The Author(s) 2014. This article is published with open access at Springerlink.com

\begin{abstract}
We construct a 2-torus homeomorphism $h$ homotopic to the identity with an attracting R. H. Bing's pseudocircle $C$ such that the rotation set of $h \mid C$ is not a unique vector. The only known examples of such an attractor were Birkhoff's attractors, arising for dissipative maps with a twist. Our construction relies heavily on the Barge-Martin method for constructing attractors as inverse limits of graphs.
\end{abstract}

\section{Introduction}

The most popular definition of an attractor is that it is a closed invariant set with isolating neighborhood. Many authors strengthen this condition by demanding that there is a point with dense orbit, that is the map is transitive when restricted to the attracting set. In other words an attractor should be in some sense indecomposable. Milnor generalized this approach even further, saying that attraction should be "observable" in the sense that there is positive probability that a point will converge to it and no subset of attractor has such a property (see [29] for a review on various concepts of attractor and evolution of this notion in the mathematical literature). Unfortunately, when it comes to the definition of strange or chaotic attractors then there is even less agreement, neither one good definition. Veerman [36] calls an attractor in $\mathbb{S}^{1} \times \mathbb{R}$ strange if it has two orbits with different (rational) rotation numbers and the associated dynamics is then referred to as rotational chaos [12]. Probably the first example of a strange attractor in the above meaning dates back to the work of Birkhoff [7] published in 1932. Roughly speaking, a Birkhoff attractor is an attractor for a (properly chosen) map

\footnotetext{
J. P. Boroński

Division of the University of Ostrava, Institute for Research and Applications of Fuzzy Modeling, National Supercomputing Centre IT4Innovations, 30. Dubna 22, 70103 Ostrava, Czech Republic e-mail: jan.boronski@osu.cz

J. P. Boroński · P. Oprocha $(\varangle)$

Faculty of Applied Mathematics, AGH University of Science and Technology, al. Mickiewicza 30, 30-059 Kraków, Poland

e-mail: oprocha@agh.edu.pl
} 
$f=\left(f_{1}, f_{2}\right): \mathbb{S}^{1} \times \mathbb{R} \rightarrow \mathbb{S}^{1} \times \mathbb{R}$ which is dissipative and satisfies twist condition, that is $\sup _{x \in \mathbb{S}^{1} \times \mathbb{R}}|\operatorname{det}(D f(x))|<1$ and $\frac{\partial}{\partial y} f_{1}(x, y)>\delta>0$, respectively. Maps of the above type, and Birkhoff attractors, received considerable attention in the mathematical literature (e.g. see [27] or [11]). An accessible summary of the results obtained in that field can be found in a book by Arrowsmith and Place [1].

The name "strange attractor" was to some extent motivated by the fact that, while plane separating, (Birkhoff) attractor with two different rotation numbers must be different from the circle, where the rotation number of a homeomorphism is always well defined and unique. Indeed, by the result of [4] any attracting cofrontier in an annulus with a non-degenerate rotation set must be indecomposable. Therefore probably the most "strange" attractor that can be expected is the pseudocircle, a peculiar continuum constructed by R.H. Bing in 1951 in [6]. It is characterized as a hereditarily indecomposable plane separating circlelike continuum. Therefore the pseudocircle is a topologically unique cofrontier. Any proper subcontinuum of the pseudocircle is a pseudoarc, the unique hereditarily indecomposable arc-like continuum $[6,25,30]$. Because of this equivalence of all subcontinua, the pseudocircle exhibits a kind of local self-similarity structure and therefore one can see it as fractal. After a decade since its construction, the pseudocircle was found to be non-homogeneous $[18,34]$, despite homogeneity of the pseudoarc. Subsequently, Kennedy and Rogers proved it to be uncountably non-homogeneous [24] in the sense, that the homeomorphism group acting on the pseudocircle has uncountably many orbits (there are uncountably many different types of points). An interesting phenomenon to point out is also the fact that the two-point compactification of the universal cover of the pseudocircle is a pseudoarc [5, Fact p.1148] but no subcontinuum of the universal covering space covers the pseudocircle. Indeed, any such subcontinuum is the pseudoarc, and the pseudoarc cannot be mapped onto the pseudocircle [33]. This peculiarity cannot be observed in "nice" spaces, such as circle or surfaces. The pseudocircle admits periodic homeomorphisms (rational rotations) that extend to orientation-preserving and orientation-reversing homeomorphisms of the annulus or 2-sphere [9,24]. It occurs also as an attracting minimal set for a $C^{\infty}$-smooth planar diffeomorphism [21,22], the minimal set of a volume-preserving planar diffeomorphism [21], or as the boundary of a Siegel disk for a holomorphic map in the complex plane [14]. It is worth mentioning that the diffeomorphism constructed in [21] can be easily modified to an annulus homeomorphism (equal to the identity on the boundary), which gives a homeomorphism of the 2-torus, with a pseudocircle as an attractor. However, this attractor is not strange (according to Veerman's definition), since it has a well-defined irrational rotation number.

In view of the aforementioned results, the following question has been known in the field of surface dynamics.

Question Is there a 2-torus homeomorphism homotopic to the identity with a pseudocircle as a strange attractor (in the sense of Veerman)?

We learned about the problem from Tobias Oertel-Jäger during Workshops on Complexity and Dimension theory of Skew Products Systems held at the Erwin Schrödinger International Institute for Mathematical Physics in Vienna, September 16 - 20, 2013 (see also [32, p.3]). The question has been known also in the context of the much more difficult Franks-Misiurewicz Conjecture [20], which states that if the rotation set on 2-torus is a line segment of positive length, then it either contains infinitely many rational vectors or it has a rational endpoint.

In the present paper we show that the pseudocircle admits the desired dynamics indeed. 
Theorem 1.1 There is a torus homeomorphism $h: \mathbb{T}^{2} \rightarrow \mathbb{T}^{2}$ homotopic to the identity, with a lift $\hat{h}: \mathbb{R}^{2} \rightarrow \mathbb{R}^{2}$, such that $h$ has an attracting pseudocircle $C$ and the rotation set $\rho(\hat{h}, C)$ is not a unique vector. Furthermore, $\left.h\right|_{C}$ is topologically mixing, has the shadowing property and positive topological entropy.

Curiously, at present time it is unknown if there are homeomorphisms of the pseudocircle with finite, non-zero entropy [31], although the homeomorphism of the pseudocircle that we construct has positive entropy. It is also not clear if pseudocircle can be nonchaotic strange attractor, i.e. strange attractor with zero topological entropy. Such a construction, if possible, cannot be obtained by an application of the results of [26] (see also [23]) which is an essential ingredient of our approach (see Lemma 3.1).

Our paper is organized as follows. Section 2 contains definitions. In Sect. 3 we first discuss the existence of a particular degree 1 circle map $f$ with non-unique rotation number. The map is modified to a circle map $g$ to preserve this dynamical property but, at the same time, to produce the pseudocircle in the inverse limit, when used as a sole bonding map. This map also induces a shift map on the inverse limit that will give the desired homeomorphism on the pseudocircle. To that end we also recall Barge and Martin's method of constructing attractors and apply it to the aforementioned inverse limit. Briefly, each factor space (circle) in the inverse limit is embedded in an annulus, and this way a new inverse limit of annuli is formed with the resulting space being the annulus. Consequently an appropriate embedding of the pseudocircle $C$ is obtained with the shift homeomorphism extending to an annulus homeomorphism $F$. In order to control the rotation number of this homeomorphism, we construct a universal covering space of the annulus as the inverse limit of universal coverings of factor spaces (annuli). This ensures that the annulus homeomorphism $F$ has a lift $\hat{F}$ with similar dynamical properties as the initially constructed circle maps $f$ and $g$. This is manifested in the fact that $\hat{F}$ has a fixed point, as well as an unbounded orbit, which ensures non-uniqueness of the rotation vector of $F$ on $C$. Finally, in Sect. 4 the torus homeomorphism with non-unique rotation vector on the pseudocircle is obtained from the annulus homeomorphism described in Sect. 3. This last section also contains some open questions for future study.

\section{Preliminaries}

\subsection{Notational convention}

In the present paper we will use the following notational convention. Coordinates of points in cartesian products will be enumerated in the subscript e.g. $\left(x_{1}, x_{2}, x_{3}, \ldots\right)$. Sets will be enumerated in the superscript e.g. $U^{1}, U^{2}, U^{3}, \ldots$ Inverse limit spaces will hold an arrow in their subscripts e.g. $X_{\leftarrow}$. Covering spaces and lifts of functions to such spaces will hold a hat e.g. $\hat{h}: \hat{\mathbb{A}} \rightarrow \hat{\mathbb{A}}$. A map will be always a continuous function. For a given space $X$, by id we will denote the identity map given by $\operatorname{id}(x)=x$ for all $x \in X$. We shall use symbols $\mathbb{R}, \mathbb{S}^{1}, \mathbb{A}, \mathbb{T}^{2}$, and $\mathbb{R}^{2}$ to denote respectively the real line, unit circle, annulus, 2-torus and the plane. For a set $B$ the symbols $\bar{B}$ and $\operatorname{int}(B)$ will denote the closure and interior of $B$ respectively. A vector in $\mathbb{R}^{2}$ will be denoted by $\left\langle a_{1}, a_{2}\right\rangle$, whereas intervals in $\mathbb{R}$ will denoted by $[a, b],(a, b),[a, b)$, and $(a, b]$. The Cartesian product of two spaces $A$ and $B$ will be denoted by $A \times B$. For infinitely many spaces $X_{1}, X_{2}, \ldots$ this product will be denoted by $\prod_{i=1}^{\infty} X_{i}$. If $X_{i}=X$ for every $i$ then we write $\prod_{i=1}^{\infty} X_{i}=X^{\mathbb{N}}$. The space of continuous self maps of a compact space $(X, d)$ is always endowed with the metric of uniform convergence 


$$
\mathfrak{d}(f, g)=\sup _{x \in X} d(f(x), g(x))
$$

which makes it a complete metric space.

\subsection{Inverse limit spaces}

Suppose a map $f: X \rightarrow X$ is given on a metric space $X$. The inverse limit space $X_{\leftarrow}=$ $\underset{\lim }{\longleftarrow}\{f, X\}$ is the space given by

$$
X_{\leftarrow}=\left\{\left(x_{1}, x_{2}, x_{3}, \ldots\right) \in X^{\mathbb{N}}: f\left(x_{i+1}\right)=x_{i}\right\} .
$$

The topology of $X_{\leftarrow}$ is induced from the product topology of $X^{\mathbb{N}}$, with the basic open sets in $X_{\leftarrow}$ given by

$$
U_{\leftarrow}=\left(f^{i-1}(U), f^{i-2}(U), \ldots, U, f^{-1}(U), f^{-2}(U), \ldots\right),
$$

where $U$ is an open subset of the $i$ th factor space $X$, and $i$ ranges over $\mathbb{N}$ (see e.g. Theorem 3 on p.79 in [16]). The map $f$ is called a bonding map. ${ }^{1}$ There is a natural homeomorphism $\sigma_{f}: X_{\leftarrow} \rightarrow X_{\leftarrow}$, called the shift homeomorphism, given by

$$
\sigma_{f}\left(x_{1}, x_{2}, x_{3}, \ldots\right)=\left(f\left(x_{1}\right), f\left(x_{2}\right), f\left(x_{3}\right), \ldots\right)=\left(f\left(x_{1}\right), x_{1}, x_{2}, \ldots\right) .
$$

It is well known that $\sigma_{f}$ preserves many dynamical properties of $f$, such as shadowing property, topological mixing and topological entropy (e.g. see [13]). It is also easy to see that if $c$ is a $p$-periodic point of $f$ then

$$
\left(f^{p-1}(c), f^{p-2}(c), \ldots, c, f^{p-1}(c), f^{p-2}(c), \ldots\right)
$$

is a $p$-periodic point of $\sigma_{f}$. For more on inverse limits see [15].

A near-homeomorphism is a surjection that is the uniform limit of a sequence of homeomorphisms. The following special case of a beautiful result due to M. Brown [10, Theorem 4] will be important to our constructions. One of the main applications of this theorem in our context, is that special types of continua can be embedded in the given space $X$ by a careful choice of map $H$.

Theorem 2.1 (Brown) Let $H: X \rightarrow X$ be a near-homeomorphism of a compact metric space $X$. Then the inverse limit space $X \leftarrow=\lim \{H, X\}$ is homeomorphic to $X$.

\subsection{Continua}

A continuum is a compact and connected metric space that contains at least two points. A continuum is arc-like (resp. circle-like) if it can be expressed as the inverse limit of arcs (resp. circles). ${ }^{2}$ A continuum is indecomposable if it cannot be expressed as the union of two proper subcontinua. It is hereditarily indecomposable if each subcontinuum is indecomposable. Among many other properties, hereditarily indecomposable continua are nowhere locally connected and contain no arcs. A cofrontier is a continuum that separates the plane into exactly two components, is the boundary of each, and none of its subcontinua is plane separating. In other words, continuum $C \subset \mathbb{R}^{2}$ is a cofrontier if $\mathbb{R}^{2} \backslash C$ has two connected

\footnotetext{
1 In general, neither the bonding map needs to be unique, nor the factor spaces in the inverse system need to be the same. However, the given definition will be sufficient for our considerations.

2 This, in general, may not need to be obtained with a single bonding map.
} 
components and $\mathbb{R}^{2} \backslash K$ has exactly one connected component for every proper subcontinuum $K \subset C$.

\subsection{Dynamical systems}

A dynamical system is a pair consisting of a continuous map $f: X \rightarrow X$ and a compact metric space $(X, d)$. By convention $f^{0}=\mathrm{id}$ and $f^{n+1}=f \circ f^{n}$ for all integers $n \geq 0$. A point $p \in X$ is periodic if $f^{n}(p)=p$ for some $n>0$. The minimal such number is called a period of $p$. If $p$ is a point of period 1 then we say that $p$ is a fixed point.

The orbit of a point $x \in X$ is the set $\operatorname{Orb}(x, f)=\left\{f^{n}(x): n \in \mathbb{N}\right\}$. The set of limit points of the orbit $\operatorname{Orb}(x, f)$ is called the $\omega$-limit set of $x$, and is denoted by $\omega(x, f)$. Similarly, for any set $U \subset X$ we denote by $\omega(U, f)$ the set of points $y$ such that there are $y_{k} \in U$ and an increasing sequence $n_{k}$ satisfying $\lim _{k \rightarrow \infty} f^{n_{k}}\left(y_{k}\right)=y$. Clearly $\omega(\{x\}, f)=$ $\omega(x, f)$.

A nonempty set $A \subset X$ is an attractor if there exists an open neighborhood $U \supset A$ such that $f(\mathrm{cl} U) \subset U$ and $\omega(U, f)=A$. Observe that by the definition, every attractor $A$ is nonempty, closed and strongly invariant (i.e. $f(A)=A$ ). We say that $f$ is (topologically) mixing if for all nonempty open sets $U, V \subset X$ there is $N$ such that $f^{n}(U) \cap V \neq \emptyset$ for every $n>N$, and (topologically) exact if for every nonempty open set $U$ there is $n>0$ such that $f^{n}(U)=[0,1]$. Clearly, each exact map is mixing but not conversely. In both cases there is a point $x \in X$ (in fact a residual set of such points) such that $\omega(x, f)=X$.

\subsection{Covering spaces}

We say that $\tau: \hat{Y} \rightarrow Y$ is a covering map if for every $y \in Y$ there is an open neighborhood $W$ of $y$ such that $\tau^{-1}(W)$ consists of pairwise disjoint sets $\left\{\hat{W}^{n}: n \in \mathbb{N}\right\}$, each of which is mapped by $\tau$ homeomorphically onto $W$; i.e. $\tau \mid \hat{W}^{n}$ is a homeomorphism. In such a case the open sets $\hat{W}^{n}$ are called sheets of $W$. A lift of a map $f: Y \rightarrow Y$ is a map $\hat{f}: \hat{Y} \rightarrow \hat{Y}$ such that $\tau \circ \hat{f}=f \circ \tau$. For simplicity, we shall also call a point $\hat{y} \in \hat{Y}$ a lift of a point $y \in Y$ if $\hat{y} \in \tau^{-1}(y)$. A universal cover $(\hat{Y}, \tau)$ of $Y$ is a cover such that if $\left(\hat{Y}^{\prime}, \tau^{\prime}\right)$ is any other cover then there is a covering map $p: \hat{Y} \rightarrow \hat{Y}^{\prime}$.

\subsection{Rotation sets}

Let $h: \mathbb{T}^{2} \rightarrow \mathbb{T}^{2}$ be a homeomorphism of the 2-torus homotopic to the identity, and let $\hat{h}: \mathbb{R}^{2} \rightarrow \mathbb{R}^{2}$ be its lift to the universal covering space $\left(\mathbb{R}^{2}, \tau\right)$. For the reminder of this article, $\tau$ will be acting in polar coordinates, that is $\tau(x, p)=\tau(y, q)$ iff $x-y, p-q \in 2 \pi \mathbb{Z}$. The same convention will be applied in the case of covering spaces of $\mathbb{S}^{1}$ and $\mathbb{A}$. The rotation set of $\hat{h}$ is given by

$$
\rho(\hat{h})=\left\{\rho \in \mathbb{R}^{2} \mid \exists z_{i} \in \mathbb{R}^{2}, n_{i} \nearrow \infty: \lim _{i \rightarrow \infty} \frac{\hat{h}^{n_{i}}\left(z_{i}\right)-z_{i}}{2 \pi n_{i}}=\rho\right\} .
$$

Note that the rotation set $\rho(\hat{h})$ is contained in the set of accumulation points of the set

$$
\left\{\frac{\hat{h}^{n}(z)-z}{2 \pi n} \mid z \in \mathbb{R}^{2}, n \in \mathbb{N}\right\}
$$


Given a subset $W$, the rotation set of $\hat{h}$ on $W$ is given by

$$
\rho(\hat{h}, W)=\left\{\rho \in \mathbb{R}^{2} \mid \exists z_{i} \in \tau^{-1}(W), n_{i} \nearrow \infty: \lim _{i \rightarrow \infty} \frac{\hat{h}^{n_{i}}\left(z_{i}\right)-z_{i}}{2 \pi n_{i}}=\rho\right\} .
$$

When $W$, in the above definition, is a singleton (say $W=\{p\}$ ) we shall just write $\rho(\hat{h}, p)$. The rotation sets of degree 1 annulus maps and circle maps are defined in an analogous way, as subsets of $\mathbb{R}$.

Note that if $h: \mathbb{A} \rightarrow \mathbb{A}$ is identity on the boundary of $\mathbb{A}$ then, identifying respective points on boundaries, we can naturally view $h$ as a map on $h: \mathbb{T}^{2} \rightarrow \mathbb{T}^{2}$ and furthermore, every $v \in \rho(h)$ is of the form $v=(a, 0)$ for some $a \in \mathbb{R}$. By the result of [4, Theorem 2.7], if $C \subset$ int $\mathbb{A} \subset R^{2}$ is a cofrontier invariant under a homeomorphism $h: \mathbb{A} \rightarrow \mathbb{A}$ and $\rho(h, C)$ does not degenerate to a unique point, then $C$ is indecomposable.

\section{Auxiliary lemmas}

Before we start our construction of a torus homeomorphism, we need to construct a map of the circle, whose inverse limit is the pseudocircle and the induced shift homeomorphism has two properly chosen periodic orbits.

Lemma 3.1 Consider a piecewise linear map $\hat{g}:[0,2 \pi] \rightarrow \mathbb{R}$ determined by the following conditions (see Fig. 1): $\hat{g}(0)=2 \pi / 3, \hat{g}(2 \pi / 3)=10 \pi / 3, \hat{g}(4 \pi / 3)=0, \hat{g}(2 \pi)=8 \pi / 3$, and $\hat{g}$ is linear on the intervals $[0,2 \pi / 3],[2 \pi / 3,4 \pi / 3]$ and $[4 \pi / 3,2 \pi]$. Extend $\hat{g}$ to a map $\hat{g}: \mathbb{R} \rightarrow \mathbb{R}$ periodically, putting $\hat{g}(x+2 \pi)=f(x)+2 \pi$. Then

(i) $\hat{g}$ is a lift to the universal cover $(\mathbb{R}, \tau)$ of a degree 1 map $g: \mathbb{S}^{1} \rightarrow \mathbb{S}^{1}$,

(ii) there is a fixed point $\hat{p}$ of $\hat{g}$ in the interval $(0,1)$,

(iii) $\hat{q}=0$ is a lift of a 3-periodic point $q$ of $g$,

(iv) the orbit of $\hat{q}$ under $\hat{g}$ is unbounded,

(v) $\rho(\hat{g}, \tau(\hat{p}))=\{0\}$ and $\rho(\hat{g}, q)=\{1 / 3\}$,

(vi) the map $g$ is topologically exact.

Proof The only statement that needs clarification is that $g$ is exact. First observe that on each interval of linearity the map $\hat{g}$ is expanding with factor at least 3 . Fix any open subset $U$ of $\mathbb{S}^{1}$ and let $\hat{I}$ be an open interval in $(0,2 \pi)$ such that $\tau(\hat{I}) \subseteq U$. Observe that if we apply $\hat{g}$ to $\hat{I}$ then there is $n \geq 0$ such that $\hat{g}^{n}(I)$ contains two critical points, that is points of the form $2 \pi k / 3$ for some $k \geq 0$ (it is because $\hat{g}$ has slope at least 3 over each interval of linearity). Then it is not hard to see that after at most 3 more iterations we will cover the whole interval $[2 \pi m, 2 \pi(m+1)]$ for some $m \in \mathbb{N}$. In other words, there exists $s>0$ such that $[2 \pi m, 2 \pi(m+1)] \subset \hat{g}^{s}(I)$. But in terms of dynamics of $g$ it means that $g^{m}(U)=\mathbb{S}^{1}$ showing that indeed $g$ is topologically exact.

The following result from [26] will allow us to produce a circle map $f$ with similar dynamical properties to the map $g$ from Lemma 3.1, but with the pseudocircle as the inverse limit space $\lim _{\longleftarrow}\left\{f, \mathbb{S}^{1}\right\}$.

Theorem 3.2 Let $\mathbb{G}$ be a topological graph and let $\mathcal{K}=\left\{a_{1}, \ldots, a_{s}\right\} \subset \mathbb{G}$ be a finite set. For every topologically exact map $g: \mathbb{G} \rightarrow \mathbb{G}$ and every $\delta>0$ there is a topologically mixing map $g_{\delta}: \mathbb{G} \rightarrow \mathbb{G}$ with the shadowing property, such that $\mathfrak{d}\left(g, g_{\delta}\right)<\delta, g_{\delta}\left(a_{j}\right)=g\left(a_{j}\right)$ for every $j$ and the inverse limit $\lim _{\longleftarrow}\left\{g_{\delta}, \mathbb{G}\right\}$ is hereditarily indecomposable. 
Fig. 1 A sketch of the graph of map $\hat{g}$ from Lemma 3.1

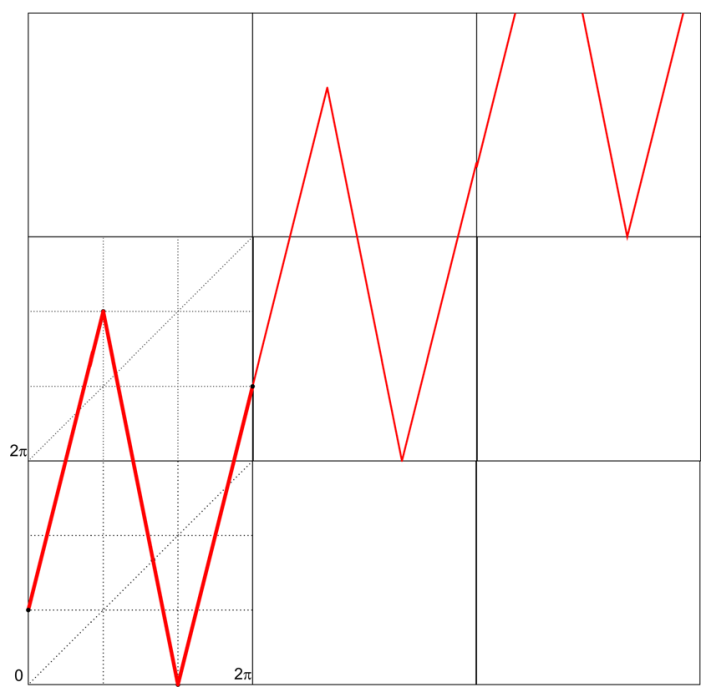

Corollary 3.3 There is a map $f: \mathbb{S}^{1} \rightarrow \mathbb{S}^{1}$, its lift $\hat{f}: \mathbb{R} \rightarrow \mathbb{R}$, a fixed point $p \in \mathbb{S}^{1}$, periodic point of period three $q \in \mathbb{S}^{1}$, and there are points $\hat{p}$ and $\hat{q}$, lifts of $p$ and $q$ respectively, such that $\hat{p}$ is a fixed point for $\hat{f}, \rho(\hat{f}, q)=\left\{\frac{1}{3}\right\}$ (in particular orbit of $\hat{q}$ is unbounded) and $\lim _{\longleftarrow}\left\{f, \mathbb{S}^{1}\right\}$ is the pseudocircle.

Proof Let $g$ be the map from Lemma 3.1 and let $\hat{g}$ be its lift to the universal cover $(\mathbb{R}, \tau)$. Let $p$ and $q$ denote the fixed point and point of period 3, respectively, provided in Lemma 3.1. Denote $\mathcal{K}=\left\{p, q, g(q), g^{2}(q)\right\}$. Without loss of generality we may assume that $\tau(0)=p$. Let $\delta>0$ be small enough, so that if $\mathfrak{o}(g, f)<\delta$ then $f$ is a map of degree 1. Let $\hat{g}$ be a lift of $g$ such that $\hat{g}(0)=0$ and let $\hat{q} \in[0,2 \pi]$ be a lift of $q$.

Let $f=g_{\delta}$ be provided by Theorem 3.2. Then $g(x)=f(x)$ for each $x \in \mathcal{K}, p$ is fixed point and $q$ is point of period 3 for both $f$ and $g$. Let $\hat{f}$ be lift of $f$ such that $\hat{f}(0)=0$. We have $\hat{g}(0)=\hat{f}(0)$ and hence we also must have $\hat{f}(q)=\hat{g}(q)$ as otherwise there is $p \in(0, q)$ such that $|\hat{f}(q)-\hat{g}(q)| \in(\delta, 1)$ which yields $\mathfrak{d}(f, g)>\delta$. By induction we obtain that $\hat{f}^{i}(\hat{q})=\hat{g}^{i}(\hat{q})$ for every $i$. The above observation implies that $\rho(\hat{f}, q)=\rho(\hat{g}, q)=\{1 / 3\}$. We remark here that $\hat{g}$ could also be obtained by a direct modification of $\hat{f}$ but it would involve another proof, analogous to the one of Theorem 3.2.

Finally, the inverse $\operatorname{limit} \lim \left\{f, \mathbb{S}^{1}\right\}$ is the pseudocircle, since $f$ has degree 1 , by the result of Fearnley [17, Theorem $\overleftarrow{6.3}$ ] (for more details see also [19] or [26, Corollary 24] and comments therein) which completes the proof.

The following lemma exhibits a special type of embedding into the annulus for a graph of a degree 1 map. This kind of an embedding will be useful in the proof of Lemma 3.7.

Lemma 3.4 Let $\hat{g}: \mathbb{R} \rightarrow \mathbb{R}$ be a lift of a map of degree 1. Then the map $\omega_{\hat{g}}:[0,2 \pi) \ni t \mapsto$ $(\hat{g}(t)(\bmod 2 \pi), \hat{g}(t)-t) \in \mathbb{S}^{1} \times \mathbb{R}$ defines a simple closed curve (Fig. 2).

Proof Denote $\alpha=\omega_{\hat{g}}([0,2 \pi))$ and observe that $\alpha$ is a closed curve since

$$
(\hat{g}(2 \pi)(\bmod 2 \pi), \hat{g}(2 \pi)-2 \pi)=(\hat{g}(0)+2 \pi(\bmod 2 \pi), \hat{g}(0)) .
$$

Next observe that if $\alpha$ has self-intersections then there are distinct $s, t \in[0,2 \pi)$ such that $(\hat{g}(t)(\bmod 2 \pi), \hat{g}(t)-t)=(\hat{g}(s)(\bmod 2 \pi), \hat{g}(s)-s)$. But then, without loss of generality, 

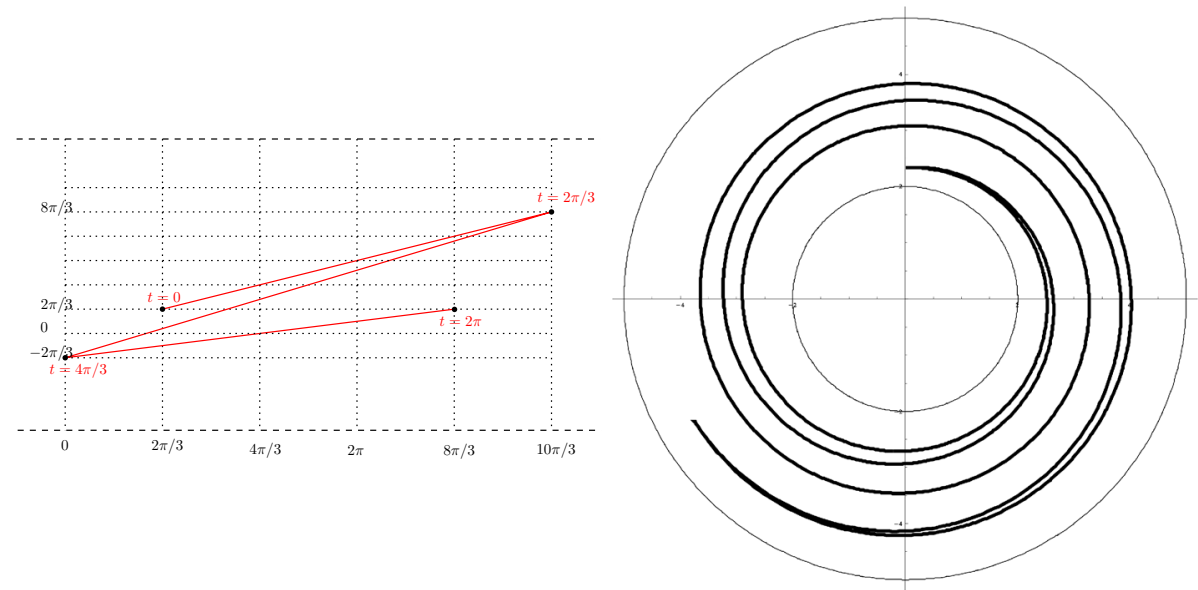

Fig. 2 The graph of the map $\omega_{\hat{g}}$ in Lemma 3.4

there is $k \in \mathbb{N}$ such that $\hat{g}(s)=\hat{g}(t)+2 \pi k$ and then $\hat{g}(t)-t=\hat{g}(s)-s=\hat{g}(t)-s+2 \pi k$. If $k=0$ then $t=s$ which is impossible, and when $k \geq 1$ then $-t \leq 0<2 \pi k-s$ which is again a contradiction, since $-t=2 \pi k-s$.

Remark 3.5 Note that if $\pi:(x, y) \mapsto(x, 0)$ and we identify $[0,2 \pi)$ with $\mathbb{S}^{1} \times\{0\}$ then the action of $\pi \circ \omega_{\hat{g}}$ coincides with the projection of $\hat{g}$ onto $\mathbb{S}^{1}$.

The following theorem summarizes a result of Barge and Martin from [2] that allows to present any arc-like continuum as a global attractor of a planar homeomorphism.

Theorem 3.6 (Barge and Martin) Every continuum $K_{\leftarrow}=\lim _{\longleftarrow}\{f,[0,1]\}$, can be embedded into a disk $D$ in such a way that

(i) $K_{\leftarrow}$ is an attractor of a homeomorphism $h: D \rightarrow D$,

(ii) $\left.h\right|_{K_{\leftarrow}}=\sigma_{f}$; i.e. $h$ restricted to $K_{\leftarrow}$ agrees with the shift homeomorphism induced by $f$, and

(iii) $h$ is the identity on the boundary of $D$.

It was pointed out in [3, p.19] that the same is true if $f$ is a degree \pm 1 circle map and $h$ is an annulus homeomorphism. Because it seems that the proof of this fact has never been published, for completeness sake we recall the procedure from [2], and adopt it to the case of degree 1 circle maps.

Lemma 3.7 Suppose $f: \mathbb{S}^{1} \rightarrow \mathbb{S}^{1}$ is of degree 1 and let $C \leftarrow=\lim \left\{f, \mathbb{S}^{1}\right\}$. Then there is an annulus homeomorphism $F: \mathbb{A} \rightarrow \mathbb{A}$ and an embedding $e: C \leftarrow \leftrightarrows \mathbb{A}$ such that

(i) $e\left(C_{\leftarrow}\right)$ is an attractor of $F$,

(ii) $\left.F\right|_{e\left(C_{\leftarrow}\right)}$ is conjugate to the shift homeomorphism $\sigma_{f}$,

(iii) $F$ is the identity on the boundary of $\mathbb{A}$.

Proof In our construction, as usual, we will be using polar coordinates, with the angular projection $\pi_{\theta}$ given by $\pi_{\theta}(r, \theta)=\theta$. For $i=1,2,3$ let $\mathbb{A}_{i}=\{(r, \theta): 4-i \leq r \leq 4+i\}$ 


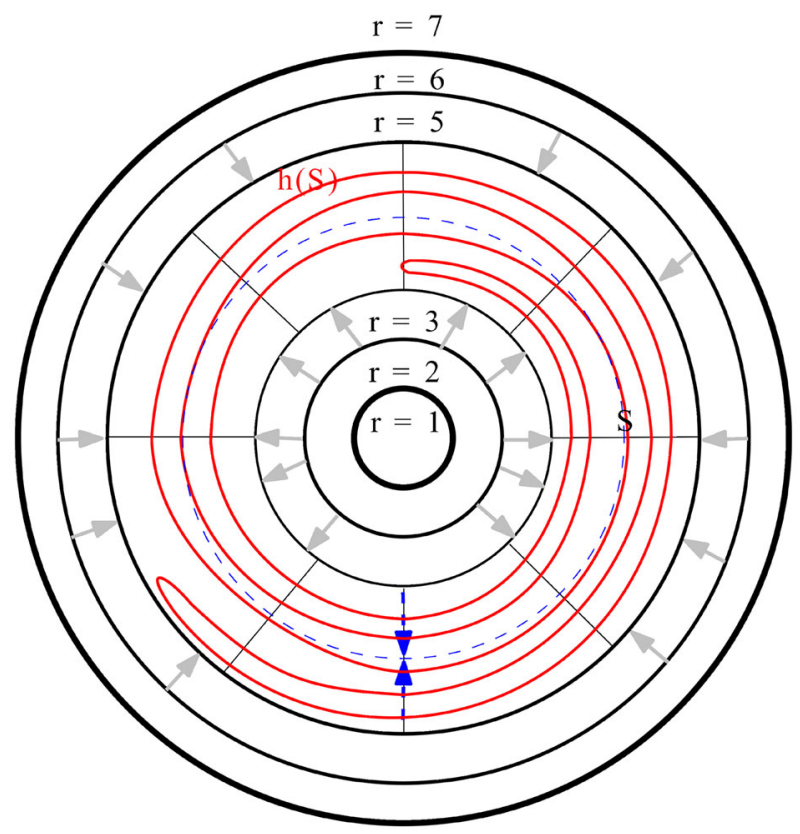

Fig. 3 The idea behind the construction of maps $G_{t}$ in Lemma 3.7

and $S=\{(r, \theta): r=4\}$. In order to define a homeomorphism $h: \mathbb{A}_{3} \rightarrow \mathbb{A}_{3}$ we first put $\left.h\right|_{\left(\mathbb{A}_{3} \backslash \mathbb{A}_{2}\right)}=$ id. Additionally, we can apply Lemma 3.4 and define $h(S)$ as a simple closed curve with the property that $\pi_{\theta}(h(r, \theta))=f(\theta)$ (i.e. after angular projection $h$ reverts to $f$ ) and $h(S) \subset$ int $\mathbb{A}_{1}$. Then, by Annulus Theorem (see [35]), $h$ can be extended to a homeomorphism of the entire annulus $\mathbb{A}_{3}$.

As the next step of our proof we will define a homotopy $G: \mathbb{A}_{3} \times[0,1] \rightarrow \mathbb{A}_{3}$, putting $G_{t}=G(\cdot, t):(r, \theta) \rightarrow\left(\eta_{t}(r), \theta\right)$ for $\left.t \in[0,1]\right\}$, where $\eta_{t}$ is a piecewise linear map defined by $\eta_{t}(1)=1, \eta_{t}(3)=3+t, \eta_{t}(5)=5-t$, and $\eta_{t}(7)=7$ (see Fig. 3 ). Observe that the following conditions are satisfied (Fig. 4):

(i) $G_{0}=\mathrm{id}$,

(ii) $G_{t}$ is a homeomorphism for each $t \neq 1$,

(iii) $\left.G_{t}\right|_{\operatorname{Bd}\left(\mathbb{A}_{3}\right)}=$ id, for all $t \in[0,1]$.

(iv) for each $\theta \in[0,2 \pi)$ we have $\{(r, \theta): 3 \leq r \leq 5\} \subseteq G_{1}^{-1}(4, \theta)$,

(v) $G_{1}\left(\mathbb{A}_{1}\right)=S$, and

(vi) for every $x \in \operatorname{int}\left(\mathbb{A}_{3}\right)$ there is $n$ such that $G_{1}^{n} \in S$.

Now we set $H=G_{1} \circ h$. By the construction, $H$ is a near-homeomorphism, hence by Theorem 2.1 it follows that $\mathbb{A}_{\leftarrow}=\lim \left\{H, \mathbb{A}_{3}\right\}$ is homeomorphic to $\mathbb{A}$. We also have a natural embedding $e: C \leftarrow \rightarrow \mathbb{A}_{\leftarrow}$ since $\overleftarrow{\left.H\right|_{S}}$ coincides with $f$ (up to identification of $S$ with $\mathbb{S}^{1}$ ). Note that if we put $U=\left\{\prod_{i=1}^{\infty}\left(r_{i}, \theta_{i}\right) \in \mathbb{A}_{\leftarrow}: 3<r_{1}<5\right\}$ then by the definition of $H$ we see that $\sigma_{H}(\bar{U}) \subset\left\{\prod_{i=1}^{\infty}\left(r_{i}, \theta_{i}\right) \in \mathbb{A}_{\leftarrow}: r_{1}=4\right\} \subset U$ and $\omega\left(U, \sigma_{H}\right) \subset \mathbb{A}_{\leftarrow} \cap S^{\mathbb{N}} \approx C_{\leftarrow}$. This shows that $C_{\leftarrow}$ is an attractor, hence the proof is completed by setting $F:=\sigma_{H}$.

In what follows we will make no distinction between the inverse limit space $C_{\leftarrow}$ and its copy $e\left(C_{\leftarrow}\right)$ embedded essentially in the annulus $\mathbb{A} \approx \mathbb{A} \leftarrow$. In the following lemma we 
Fig. 4 Graph of the map $\eta_{t}$

7

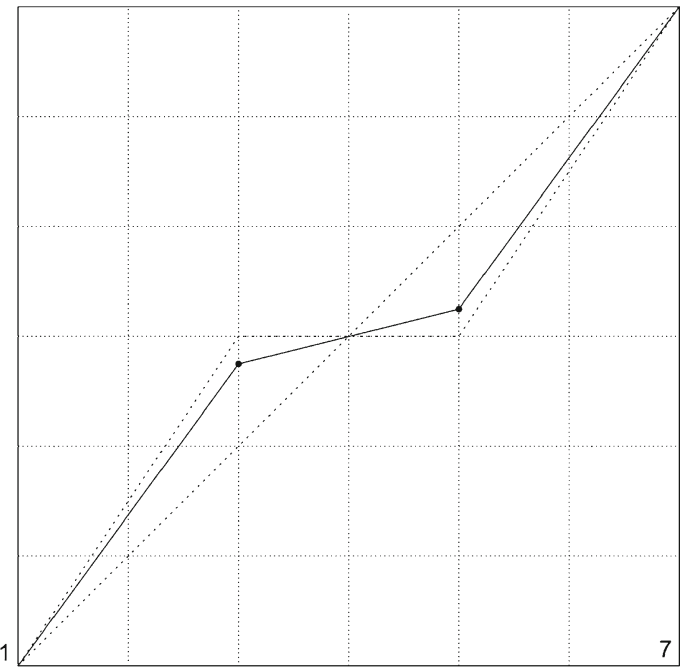

show how a lift of a circle map $f$ can be related to a lift of the annulus homeomorphism $F$ constructed in the Lemma 3.7.

Lemma 3.8 Let $f: \mathbb{S}^{1} \rightarrow \mathbb{S}^{1}, \hat{f}: \mathbb{R} \rightarrow \mathbb{R}$ be the maps from Corollary 3.3 and $F: \mathbb{A} \leftarrow \rightarrow$ $\mathbb{A}_{\leftarrow}$ be the coresponding annulus homeomorphism from Lemma 3.7. There is a universal $\operatorname{cover}\left(\hat{\mathbb{A}}_{\leftarrow}, \tau_{\leftarrow}\right)$ of $\mathbb{A}_{\leftarrow}$ and a lift $\hat{F}: \hat{\mathbb{A}}_{\leftarrow} \rightarrow \hat{\mathbb{A}}_{\leftarrow}$ of $F$ with the property $\left.\hat{F}\right|_{\tau^{-1}\left(C_{\leftarrow}\right)}=\sigma_{\hat{f}}$.

Proof Let $h: \mathbb{A}_{3} \rightarrow \mathbb{A}_{3}$ and $G_{t}: \mathbb{A}_{3} \rightarrow \mathbb{A}_{3}$ for every $t \in[0,1]$ be as in the proof of Lemma 3.7 and $(\hat{\mathbb{A}}, \tau)$ be a universal cover of $\mathbb{A}_{3}$. Let $p \in S$ be the fixed point of $f=\left.H\right|_{S}$ provided by Corollary 3.3, where as we remember $H=G_{1} \circ h$. Fix $\hat{p} \in \tau^{-1}(p)$. There are unique lifts $\hat{h}: \hat{\mathbb{A}} \rightarrow \widehat{\mathbb{A}}$ and $\hat{G}_{t}: \hat{\mathbb{A}} \rightarrow \widehat{\mathbb{A}}$, of $h$ and $G_{t}$ respectively (where $t \in[0,1]$ ), such that $\hat{h}(\hat{p})=\hat{G}_{t}(\hat{p})=\hat{p}$ for every $t \in[0,1]$. In addition $\hat{h}$ and $\hat{G}_{t}$ are homeomorphisms for every $t \in[0,1)$. Setting $\hat{H}=\hat{G}_{1} \circ \hat{h}$ we obtain a lift of $H$ which is also a near-homeomorphism.

Let $\hat{\mathbb{A}}_{ \pm \infty}=\hat{\mathbb{A}} \cup\{-\infty, \infty\}$ be a two-point compactification of $\widehat{\mathbb{A}}$ by $-\infty$ and $\infty$. Note that $\hat{\mathbb{A}}_{ \pm \infty}$ is topologically a disk. Since $\hat{h}$ and $\hat{G}_{t}$ for every $t \in[0,1]$ are homotopic to the identity, they extend uniquely to $\hat{\mathbb{A}}_{ \pm \infty}$. This is simply obtained by setting $\hat{h}(\infty)=\hat{G}_{t}(\infty)=\infty$ and $\hat{h}(-\infty)=\hat{G}_{t}(-\infty)=-\infty$ for every $t \in[0,1]$. Then the extended map $\hat{H}: \hat{\mathbb{A}}_{ \pm \infty} \rightarrow \hat{\mathbb{A}}_{ \pm \infty}$ is a near-homeomorphism and it is the identity on the boundary of the disc $\hat{\mathbb{A}}_{ \pm \infty}$. Now consider the inverse limit space $D_{\leftarrow}=\lim \left\{\hat{\mathbb{A}}_{ \pm \infty}, \hat{H}\right\}$. The space $D_{\leftarrow}$ is a disk, by Theorem 2.1, and if we denote $-\hat{\infty}=(-\infty,-\infty,-\infty, \ldots)$ and $\hat{\infty}=(\infty, \infty, \infty, \ldots)$ then $\hat{\infty}$ and $-\hat{\infty}$ are on the boundary of $D_{\leftarrow}$. In particular $D_{\leftarrow}$ is simply connected. Let $\tau_{\leftarrow}: D_{\leftarrow} \backslash\{-\hat{\infty}, \hat{\infty}\} \rightarrow \mathbb{A}_{\leftarrow}$ be given by $\tau_{\leftarrow}\left(x_{1}, x_{2}, x_{3}, \ldots\right)=\left(\tau\left(x_{1}\right), \tau\left(x_{2}\right), \tau\left(x_{3}\right), \ldots\right)$. Recall that $\mathbb{A}_{\leftarrow}=\lim \left\{H, \mathbb{A}_{3}\right\}$.

We claim that $\hat{\mathbb{A}}_{\leftarrow}=D_{\leftarrow} \backslash\{-\hat{\infty}, \hat{\infty}\}$ is a universal cover of $\mathbb{A}_{\leftarrow}$ with $\tau_{\leftarrow}$ as the covering map. Since $D_{\leftarrow} \backslash\{-\hat{\infty}, \hat{\infty}\} \cong \mathbb{R} \times[0,1]$, we only need to show that $\tau_{\leftarrow}$ is a covering map.

Note that

$$
\tau \circ \hat{H}=H \circ \tau \text {. }
$$

Since $\tau$ is a covering map, there is a disk neighborhood $U$ of $x_{1}$ such that $\tau^{-1}(U)=$ $\bigcup\left\{\hat{U}^{n}: n \in \mathbb{N}\right\}$, where all sets $\hat{U}^{n}$ are pairwise disjoint, each $\hat{U}^{n}$ is open in $\hat{\mathbb{A}}$ and mapped homeomorphically onto $U$ by $\tau$, i.e. $\hat{U}^{n}$ are the sheets of $U$. We shall show that, for a fixed $n$, the set $\hat{U}_{\leftarrow}^{n}=\left(\hat{U}^{n}, \hat{H}^{-1}\left(\hat{U}^{n}\right), \hat{H}^{-2}\left(\hat{U}^{n}\right), \ldots\right)$ is a sheet of 
$U_{\leftarrow}=\left(U, H^{-1}(U), H^{-2}(U), \ldots\right)$. Note that $\hat{U}_{\leftarrow}^{n}$ is open, as it is a basic set for the topology of $\mathbb{A}_{\leftarrow}$. By (3.1) it should be clear that $\tau_{\leftarrow}\left(\hat{U}_{\leftarrow}^{n}\right)=U_{\leftarrow}$ for each $n \in \mathbb{N}$ and $\tau_{\leftarrow}^{-1}\left(U_{\leftarrow}\right)=\bigcup\left\{\hat{U}_{\leftarrow}^{n}: n \in \mathbb{N}\right\}$. It remains to show that $\tau_{\leftarrow} \mid \hat{U}_{\leftarrow}^{n}$ is injective for every $n \in \mathbb{N}$.

We will prove by induction that $\tau$ is injective on $\hat{H}^{-k}\left(\hat{U}^{n}\right)$ for every $k \geq 0$. For $k=0$ there is nothing to prove, so assume that $\tau$ is injective on $\hat{H}^{-k}\left(\hat{U}^{n}\right)$ but not on $\hat{H}^{-k-1}\left(\hat{U}^{n}\right)$ for some $k \geq 0$. Take any distinct $x, y \in \hat{H}^{-k-1}\left(\hat{U}^{n}\right)$ such that $\tau(x)=\tau(y)$. Then $x=y+v$ for some $v \in 2 \pi \mathbb{Z}$ and $\hat{H}(x)=\hat{H}(y+v)=\hat{H}(y)+v$ since $H$ is of degree 1 . But then by (3.1) and the fact that $\hat{H}(x), \hat{H}(y) \in \hat{H}^{-k}\left(\hat{U}^{n}\right)$ we obtain that

$$
H(\tau(x))=\tau(\hat{H}(x)) \neq \tau(\hat{H}(y))=H(\tau(y))=H(\tau(x))
$$

which is a contradiction. But this shows that

$$
\tau \leftarrow \hat{U}_{\leftarrow}^{n}=\left(\left.\tau\right|_{\hat{U}^{n}},\left.\tau\right|_{\hat{H}^{-1}\left(\hat{U}^{n}\right)},\left.\tau\right|_{\hat{H}^{-2}\left(\hat{U}^{n}\right)}, \ldots\right)
$$

is injective and so the claim is proved.

The proof is completed by putting $\hat{F}:=\sigma_{\hat{H}}$.

In the following lemma we relate the rotation set of $f$ to the rotation set of $F$. In the proof, we use the same notation as in the proof of the previous lemma (Fig. 5).

Lemma 3.9 Let $f, \hat{f}, F$ and $\hat{F}$ be as in Lemma 3.8. Then the rotation set of $\hat{F}$ contains two distinct numbers.

Proof Recall that $p$ is a fixed point of $f$ with $\rho(\hat{f}, p)=\{0\}$, and $q$ is a 3-periodic point of $f$ with $\rho(\hat{f}, q)=\left\{\frac{1}{3}\right\}$. For any $\hat{q} \in \tau^{-1}(q)$ we have

$$
\lim _{k \rightarrow \infty} \hat{f}^{k}(\hat{q})=\infty
$$

As a consequence

$$
\lim _{k \rightarrow \infty} \sigma_{\hat{f}}^{k}\left(\hat{q}, \hat{H}^{-1}(\hat{q}), \hat{H}^{-2}(\hat{q}), \ldots\right)=\lim _{k \rightarrow \infty} \hat{F}^{k}\left(\hat{q}, \hat{H}^{-1}(\hat{q}), \hat{H}^{-2}(\hat{q}), \ldots\right)=\hat{\infty}
$$

This shows that $\rho(\hat{F}, p)=\{0\}$, whereas $\rho(\hat{F}, q) \neq\{0\}$. Indeed, since the orbit $\left\{\hat{F}^{n}(\hat{q})\right.$ : $n=1,2,3, \ldots\}$ is unbounded, and $q$ is 3-periodic for $F$, there must be an integer $k \neq 0$ such that $\hat{F}^{3}(\hat{q})=\hat{q}+2 k \pi$. Consequently $\rho(\hat{F}, q)=\left\langle\frac{k}{3 n}, 0\right\rangle$.

Combining all the previous facts together we get the following:

Theorem 3.10 There is an annulus homeomorphism $F: \mathbb{A} \rightarrow \mathbb{A}$, its lift to the universal cover $\hat{F}$ and a pseudocircle $C \subseteq \mathbb{A}$ such that

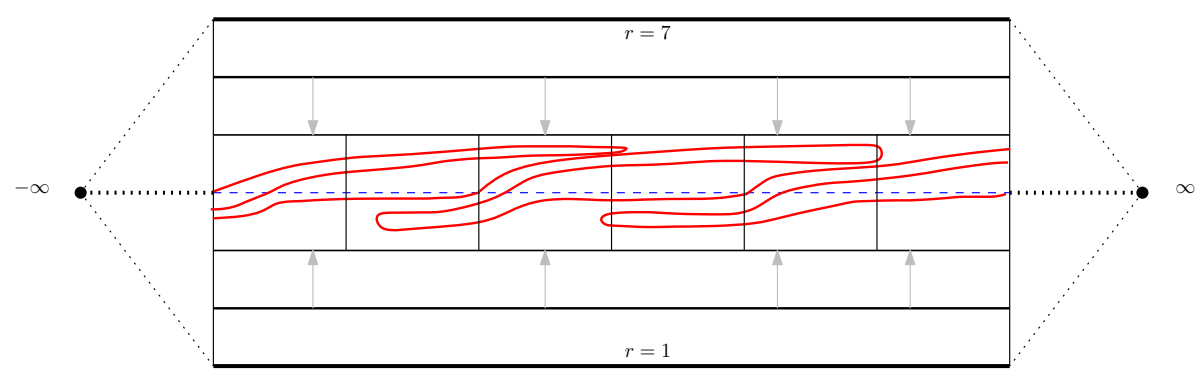

Fig. 5 Approximation in the universal cover 
(i) $F(C)=C$,

(ii) $C$ is an attractor of $F$,

(iii) the rotation set of $\rho(\hat{F}, C)$ contains two distinct numbers (one of which is 0 ),

(iv) $F$ is the identity on the boundary components.

Remark 3.11 Note that the annulus homeomorphism $F$ above has an $m$-periodic point in $C$ for every $m \geq 3$. All such periodic points are induced from the circle map $f$, for which they are guaranteed by the period-forcing result of Block [8, Theorem A].

\section{Main result}

Theorem 1.1. There is a torus homeomorphism $h: \mathbb{T}^{2} \rightarrow \mathbb{T}^{2}$ homotopic to the identity, with a lift $\hat{h}: \mathbb{R}^{2} \rightarrow \mathbb{R}^{2}$, such that $h$ has an attracting pseudocircle $C$ and the rotation set $\rho(\hat{h}, C)$ is not a unique vector. Furthermore, $\left.h\right|_{C}$ is topologically mixing, has shadowing property and positive topological entropy.

Proof Let $\mathbb{A} \rightarrow \mathbb{A}$ be the annulus homeomorphism from Theorem 3.10 with the attracting pseudocircle $C$. Identify two points in $\mathbb{A}$ if they are on the boundary of $\mathbb{A}$ and share the angular coordinates. That is, let $\mathbb{A}=\{(r, \rho) \mid 1 \leq r \leq 2\}$ be given in polar coordinates and consider a relation $\sim$ on $\mathbb{A}$ defined by

$$
\left(r_{1}, \rho_{1}\right) \sim\left(r_{2}, \rho_{2}\right) \quad \text { if and only if }\left(r_{1}=r_{2}-1 \text { or } r_{2}=r_{1}-1\right) \text { and } \rho_{1}=\rho_{2} .
$$

Then $\mathbb{A} / \sim$ is homeomorphic to $\mathbb{T}^{2}$, and $F$ induces a homeomorphism $h: \mathbb{T}^{2} \rightarrow \mathbb{T}^{2}$. The lift $\hat{F}$ provided by Theorem 3.10 gives a lift $\hat{h}$ of $h$ to the universal covering space with two rotation vectors $\langle 0,0\rangle,\left\langle\frac{k}{3 n}, 0\right\rangle \in \rho(\hat{h}, C)$. Finally, $\left.h\right|_{C}$ is topologically mixing, has shadowing property and positive topological entropy since $\left.h\right|_{C}=\sigma_{f}$ and $f$ has all these properties.

It should be clear that our procedure of producing a connected attractor with rotation interval will also work for other degree 1 circle maps that have two distinct numbers in their rotation set. For example, the map $g$ from Lemma 3.1 produces an attractor with non-unique rotation vector that is not homeomorphic to the pseudocircle. Indeed, one easily see that the attractor $A_{g}=\lim _{\{}\left\{\mathbb{S}^{1}, g\right\}$ contain arcs (the same seems true for Birkhoff attractors [27]), hence it is topologically distinct from the pseudocircle that does not contain any arcs. This motivates the following two problems.

Question 1 Are there uncountably many topologically distinct connected strange attractors in $\mathbb{T}^{2}$ ?

Question 2 Characterize the inverse limits $\lim \left\{\mathbb{S}^{1}, f\right\}$ for degree 1 circle maps $f$, that admit two distinct rotation numbers. What conditions on such $f: \mathbb{S}^{1} \rightarrow \mathbb{S}^{1}$ and $g: \mathbb{S}^{1} \rightarrow \mathbb{S}^{1}$ assure that $\lim _{\longleftarrow}\left\{\mathbb{S}^{1}, f\right\}$ and $\lim _{\longleftarrow}\left\{\mathbb{S}^{1}, g\right\}$ are topologically distinct?

It is expected that, as in the case of Buckethandle Continua [37], the number of turning points of the bonding map will determine non-homeomorphic classes of continua. For example a piecewise monotone bonding map $g_{5}$ built on a 5-periodic orbit will lead to an inverse limit space topologically distinct from the one obtained by using the map $g$ in Lemma 3.1.

As a reminder, we also repeat a question stated before in the introduction.

Question 3 Can the pseudocircle $C$ be a strange non-chaotic attractor in $\mathbb{T}^{2}$ (i.e. dynamics on $C$ has zero entropy and non-unique rotation vector)? 
Finally, we wonder if pseudocircles can arise as approximations of plane separating attractors for annulus homeomorphisms whose rotation set is nondegenerate.

Question 4 Suppose $h$ is an annulus homeomorphism with an attracting circle-like cofrontier $\Lambda$ such that the rotation set of $h \mid \Lambda$ is nondegenerate. Given $\epsilon>0$, is there an annulus homeomorphism $g$ and a pseudocircle $C$, such that $\mathfrak{d}(h, g)<\epsilon$ and the rotation set of $g \mid C$ is nondegenerate?

The above question is motivated by the fact that by Theorem 3.2 every topologically exact map on $\mathbb{S}^{1}$ can be approximated by a map that, when used as a sole bonding map of an inverse system, gives the pseudocircle in the inverse limit. In fact the same is true for the identity map on $\mathbb{S}^{1}$ according to Remark on p. 209 in [23]. Therefore it seems that if $\Lambda$ is circle-like and $h$ is constructed by the inverse limit technique considered in the present paper, then the above question has an affirmative answer, but the problem may be more difficult to solve in its full generality. Also, it may be interesting to determine if pseudocircles could approximate other attracting cofrontiers admitting annulus homeomorphisms with non-unique rotation number, that are not necessarily circle-like. This is motivated by the fact that the pseudoarc approximates any continuum in $\mathbb{R}^{n}$, for $n>1$ and every map between two subcontinua in $\mathbb{R}^{m}$, with $m>2$ can be approximated by a homeomorphism between two pseudoarcs [28].

Acknowledgments The authors express many thanks to Henk Bruin, Tobias Oertel-Jäger, Alejandro Passeggi and Sonja Štimac for stimulating discussions during Workshops on Complexity and Dimension theory of Skew Products Systems held at the Erwin Schrödinger International Institute for Mathematical Physics (ESI) in Vienna, September 16-20, 2013. The authors are grateful for the kind hospitality of the institute during their stay in Vienna supported by ESI, the DFG network Grant Oe 538/3-1, OeAD (Project PL 02/2013) and MNiSW (Project AT 2/2013-15). J. Boroński's work was supported by the European Regional Development Fund in the IT4Innovations Centre of Excellence project (CZ.1.05/1.1.00/02.0070). J. Boroński also gratefully acknowledges the partial support from the MSK DT1 Support of Science and Research in the Moravian-Silesian Region 2013 (RRC/05/2013). The research of P. Oprocha was supported by the Polish Ministry of Science and Higher Education from sources for science in the years 2013-2014, Grant No. IP2012 004272.

Open Access This article is distributed under the terms of the Creative Commons Attribution License which permits any use, distribution, and reproduction in any medium, provided the original author(s) and the source are credited.

\section{References}

1. Arrowsmith, D.K., Place, C.M.: An Introduction to Dynamical Systems. Cambridge University Press, Cambridge (1990)

2. Barge, M., Martin, J.: The construction of global attractors. Proc. Am. Math. Soc. 110, 523-525 (1990)

3. Barge, M., Roe, R.: Circle maps and inverse limits. Topol. Appl. 36, 19-26 (1990)

4. Barge, M., Gillette, R.: Rotation and periodicity in plane separating continua. Ergod. Theory Dyn. Sys. 11, 619-631 (1991)

5. Bellamy, D.P., Lewis, W.: An orientation reversing homeomorphism of the plane with invariant pseudoarc. Proc. Am. Math. Soc. 114(4), 1145-1149 (1992)

6. Bing, R.H.: Concerning hereditarily indecomposable continua. Pac. J. Math. 1, 43-51 (1951)

7. Birkhoff, G.D.: Sur quelques curves fermeé remarkable. Bull. Soc. Math. France 60, 1-26 (1932)

8. Block, L.: Periodic orbits of continuous mappings of the circle. Trans. Am. Math. Soc. 260, 553-562 (1980)

9. Boroński, J.P.: Periodic orientation reversing homeomorphisms of the 2-sphere with an invariant pseudocircle. Topol. Proc. 40, 83-90 (2012)

10. Brown, M.: Some applications of an approximation theorem for inverse limits. Proc. Am. Math. Soc. 11, 478-483 (1960)

11. Casdagli, M.: Periodic orbits for dissipative twist maps. Ergod. Theory Dyn. Syst. 7, 165-173 (1987) 
12. Casdagli, M.: Rotational chaos in dissipative systems. Phys. D 29, 365-386 (1988)

13. Chen, L., Li, S.H.: Dynamical connections between a continuous map and its inverse limit space. IN: Continuum Theory and Dynamical Systems, Lecture Notes in Pure and Applied Mathematics, vol 149, pp 89-97, Dekker, New York (1993)

14. Chéritat, A.: Relatively compact Siegel disks with non-locally connected boundaries. Math. Ann. 349, 529-542 (2011)

15. Eilenberg, S., Steenrod, N.: Foundations of Algebraic Topology. Princeton University Press, Princeton (1952)

16. Engelking, R.: Zarys topologii oglnej. (Polish) [Outline of general topology] Biblioteka Matematyczna. Tom 25 Pastwowe Wydawnictwo Naukowe, Warsaw (1965)

17. Fearnley, L.: The pseudo-circle is unique. Trans. Am. Math. Soc. 149, 45-64 (1970)

18. Fearnley, L.: The pseudo-circle is not homogeneous. Bull. Am. Math. Soc. 75, 554-558 (1969)

19. Fearnley, L.: Classification of all hereditarily indecomposable circularly chainable continua. Trans. Am. Math. Soc. 168, 387-401 (1972)

20. Franks, J., Misiurewicz, M.: Rotation sets of toral ows. Proc. Am. Math. Soc. 109, 243-249 (1990)

21. Handel, M.: A pathological area preserving $C^{\infty}$ diffeomorphism of the plane. Proc. Am. Math. Soc. 86, 163-168 (1982)

22. Herman, M.: Construction of some curious diffeomorphisms of the Riemann sphere. J. Lond. Math. Soc. (2) 34, 375-384 (1986)

23. Kawamura, K., Tuncali, H.M., Tymchatyn, E.D.: Hereditarily indecomposable inverse limits of graphs. Fund. Math. 185, 195-210 (2005)

24. Kennedy, J., Rogers Jr, J.T.: Orbits of the pseudocircle. Trans. Am. Math. Soc. 296, 327-340 (1986)

25. Knaster, B.: Un continu dont tout sous-continu est indécomposable. Fund. Math. 3, 247-286 (1922)

26. Kościelniak, P., Oprocha, P., Tuncali, M.: Hereditary indecomposable inverse limits of graphs: shadowing, mixing and exactness. Proc. Am. Math. Soc. 142, 681-694 (2014)

27. Le Calvez, P.: Propriétés des attracteurs de Birkhoff. Ergod. Theory Dyn. Syst. 8(2), 241-310 (1988)

28. Lewis, W.: Most maps of the pseudo-arc are homeomorphisms. Proc. Am. Math. Soc. 91(1), 147-154 (1984)

29. Milnor, J.: On the concept of attractor. Commun. Math. Phys. 99, 177-195 (1985)

30. Moise, E.E.: An indecomposable plane continuum which is homeomorphic to each of its nondegenerate subcontinua. Trans. Am. Math. Soc. 63, 581-594 (1948)

31. Mouron, C.: A chainable continuum that admits a homeomorphism with entropy of arbitrary value. Houst. J. Math. 35, 1079-1090 (2009)

32. Oertel-Jäger, T., Passeggi, A.: On torus homeomorphisms semiconjugate to irrational rotations. Ergod. Theory Dyn. Syst. pp 1-24 (online). doi:10.1017/etds.2014.23

33. Rogers Jr, J.T.: Mapping the pseudo-arc onto circle-like, self-entwined continua. Mich. Math. J. 17, 91-96 (1970)

34. Rogers Jr, J.T.: The pseudo-circle is not homogeneous. Trans. Am. Math. Soc. 148, 417-428 (1970)

35. Rolfsen, D.: Knots and links. In: Mathematics Lecture Series. No. 7. Publish or Perish, Inc, Berkeley (1976)

36. Veerman, P.: Strange attractors in dissipative maps with one angular variable. In: European Conference on Iteration Theory (Caldes de Malavella, 1987), pp. 338-362. World Scientific, Teaneck, NJ (1987)

37. Watkins, W.T.: Homeomorphic classification of certain inverse limit spaces with open bonding maps. Pac. J. Math. 103, 589-601 (1982) 